ASM Sc. J., 13, 2020

https://doi.org/10.32802/asmscj.2020.sm26(2.21)

\title{
The Transition from Real Numbers to Complex Numbers
}

\author{
Elvent Taliban ${ }^{1 *}$, Kin Eng Chin ${ }^{2}$ and Fui Fong Jiew ${ }^{3}$ \\ ${ }^{1}$ Faculty of Psychology \& Education, Universiti Malaysia Sabah, Kota Kinabalu, Sabah, Malaysia \\ ${ }^{2}$ College of Education, Psychology \& Social Work, Flinders University, South Australia, Australia \\ ${ }^{3}$ School of Teacher Education and Leadership, Faculty of Education, Queensland University of Technology, \\ Queensland, Australia
}

\begin{abstract}
Humans make sense of mathematics by relating it to personal conceptions and experiences. This might not be a smooth process as it may involve conceptions that work in an old context but do not work in a new context. The framework proposed by Chin and Tall (2012), Chin (2013) and Chin (2014) highlights how prior experiences shape humans' conceptions can be either supportive or problematic in making sense of extended contexts of mathematics. A supportive conception is a conception that supports learning and generalisation in a new context while a problematic conception is a conception that impedes learning and generalisation in a new context. By employing this framework, this study aims to explore how a group of respondents that consists of 30 mathematics undergraduate students from a public university in Malaysia make sense of complex numbers. The system of complex numbers is an extension of real numbers thus students will learn real numbers prior to learning complex numbers. How the respondents cope with the transition from real numbers context to complex numbers context is the focus of this study. Data were collected through a questionnaire and follow up interviews. Respondents participated in the follow up interviews voluntarily and data from three respondents were reported in this study. The result shows that these respondents have several supportive conceptions, such as the concept of addition and multiplication of real variable. The data also support the existence of problematic conceptions in making sense of complex numbers, namely the concept of positive-negative numbers and inequalities. These conceptions are originated from the context of real numbers.
\end{abstract}

Keywords: complex numbers, problematic conceptions, real numbers, supportive conceptions

\section{INTRODUCTION}

The process of sense making in mathematics often involves the transition of different contexts. Chin and Tall (2012) proposed the idea of supportive and problematic conceptions in the sense making of trigonometry. Supportive conceptions support learners to make sense of new contexts while problematic conceptions impede learning. This paper extends their idea and focuses on the conceptions involve in the transition from real numbers to the complex numbers. There are several conceptions that have been identified related directly to the transition from real numbers to complex numbers. These conceptions were grouped either as supportive or problematic conceptions. Speaking of the supportive conception, Conner, Rasmussen, Zandieh and Smith (2007) said that students are able to perform addition and multiplication of complex numbers and have expressed a moderate comfort level with basic complex arithmetic. It is evident that supportive conceptions will help learners to generalize their knowledge in a new context. In light of the problematic conception, Tirosh and Almog (1989) (as cited in Danenhower, 2000) found out that students had difficulty understanding the concept of inequalities in relation to the

*Corresponding author's e-mail: elitesquad89@gmail.com 
real number system which does not extend to the complex number system. This finding is also supported by Danenhower (2000). Based on the previous studies, it is evident that the supportive and problematic conceptions are the norm in the process of making sense of mathematics. Hence, this study is conducted to find the root causes of a problematic conception to help alleviate the issue of its occurrences which hinders the learners learning experience. A recent research by Chin and Jiew (2018) has indicated that university students have problematic conceptions that impede sense making of complex numbers in particular on conceiving $\sqrt{-1}$ as a single entity.

Supportive conceptions emerge when previously learned concepts match with newly learned concepts. Take for instance, the conception that a longer number has a greater value might arise in the context of positive integers. However, in the context of decimals, this conception might become a problematic conception because a longer number might not have a greater value. As an illustration, 1.256 is longer than 1.35 but 1.35 has a greater value in this case. This shows that learners may be impeded by problematic conceptions in making sense of concepts in new contexts. A pictorial representation of this framework is shown in Chin and Jiew (2019) and this framework is further refined in Chin and Pierce (2019). In short, a supportive conception may be regarded as a conception that works in an old context and continues to work in a new context. On the other hand, a problematic conception may be considered as a conception that works in an old context but does not continue to work in a new context.

\section{METHODOLOGY}

This is an empirical study which involved 30 undergraduate mathematic students from a public university in Malaysia. The questionnaire was first distributed to the respondents then 5 of them volunteered to take part in the interviews. Due to the space constraint, this study reported data only from 3 respondents as their responses were able to represent the whole group of respondents. They were interviewed chronologically from item 1 to item 18 to gain more insight on their written responses in the questionnaire. The duration of the followup interviews, on average was 50 minutes per respondent and each follow-up interview was a one to one session. In this study, the Quasi-Judicial method of analysis was adopted in analysing the data that have been collected. This method of analysis is unique when compared to other methods of qualitative data analysis because, rather than letting the theories emerge from data, this method uses the existing data to test pre-existing theories (Bromley, 1986, 1990).

The data of this study were collected through a set of questionnaires consisting of eighteen mathematical items related to complex numbers. Then follow-up interviews were conducted with the respondents in order to gain more insight on their thinking. These respondents have already taken courses related to complex numbers. After a close examination of all the received responses, the responses of three respondents for five mathematical items were chosen and reported in this section. This was because they showed qualitatively different responses and could cover the spectrum of responses of this group of respondents. Respondent 1 is a second-year female student with a CGPA of 3.43. Respondent 2 is a second-year female student with a CGPA of 3.20. Respondent 3 is a second-year male student with the CGPA of 3.47. Items 7, 10 and 11 were chosen to show the respondents' problematic conceptions while items 17 and 18 were used to demonstrate the supportive conceptions of the respondents. The selected items to be reported in this paper are as follows:

(1) Given a complex number $z=-2-i$. Do you think $\mathrm{z}$ is a negative complex number? Explain your answer.

(2) Do you think that $2+3 i<4+3 i$ ?

(3) Is the working shown correct? Explain.

$$
\begin{aligned}
& \frac{2}{i}=\frac{\sqrt{4}}{\sqrt{-1}}=\sqrt{\frac{4}{-1}} \\
& =\sqrt{-4}=\sqrt{-1} \sqrt{4} \\
& =2 i
\end{aligned}
$$

(4) Two complex numbers $z=2+3 i$ and $u=6+2 i$. Find the value of $z+u$.

(5) Two complex numbers $z=2-4 i$ and $u=3+2 i$. Find the value of $z \cdot u$.

\section{RESULTS \& DISCUSSIONS}

In presenting the follow-up interview excerpts, we represent the researcher as $\mathrm{R}$, Respondent 1 as $\mathrm{R} 1$, Respondent 2 as R2 and Respondent 3 as R3. 
Item 7 in the questionnaire asked "Given a complex number $z=-2-i$. Do you think $z$ is a negative complex number? Explain your answer.”

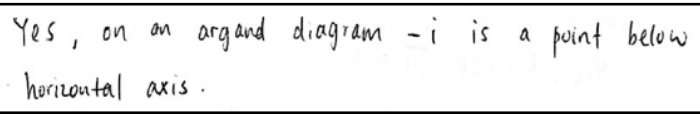

Figure 1. Respondent 1 (Item 7)

$\mathrm{R}: \quad$ You were given $z=-2-i$, do you think that $\mathrm{z}$ is a negative complex numbers?

R1: Yes. Because there is a negative ithere (pointing to the negative sign) and then it is below the horizontal axis.

$\mathrm{R}$ : Can you draw a diagram?

R1 : (Drawing an argand diagram and plotting the complex numbers $\mathrm{z}$ on the third quadrant).

Respondent 1was trying to make sense of a negative complex number by referring back to her previous experience in dealing with real numbers. In real numbers, a negative number is a number with a negative sign such as $-2,-0.8$ and so on. She noticed a negative sign attached to the complex number thus she immediately conceived that the given complex number was a negative complex number. This conception might have arisen based on her experience in working with real numbers. Building on this problematic conception then she came up with her conclusion. She knew that the complex number was a point on the complex plane, and even sketched an argand diagram for $-2-i$. She stressed that the number is a negative complex number because the plotted point is below the horizontal axis. She thought that $-2-i$ was negative because it was below the horizontal axis. On the other hand, Respondent 2 responded differently for this.

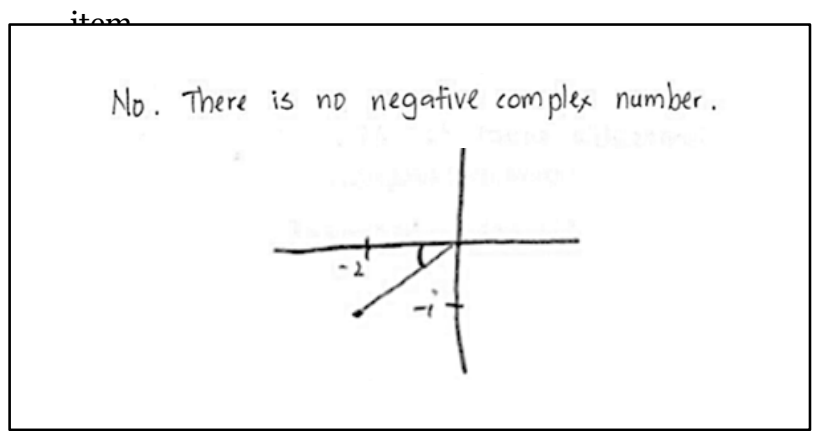

Figure 2: Respondent 2 (Item 7)

$\mathrm{R}: \quad$ Given a complex number $=-2-i$, Do you think $\mathrm{z}$ is a negative complex number?

R2 : Actually I don't understand what is negative complex numbers.

R : Okay, for example, just like in real numbers we have positive and negative number such as negative two, positive two. So, is that the same case in complex numbers?

R2: I think no, because in complex numbers what I learned before I didn't see the negative complex numbers. Because negative errr... we have the argument $\mathrm{z}$ for the angle ...

R : $\quad$ Okay...

R2: We have the distance part. I never see negative complex numbers. because like this one $z$ equals to negative two minus $i$ is a left down (trying to imagine)

$\mathrm{R}$ : $\quad$ You want to draw?

R2 : It's the third quadrant?

$\mathrm{R}$ : $\quad$ You can draw the diagram if you want.

R2 : (Starting to draw a diagram (see Figure 2)) So we can only count this angle or this distance (pointing to the diagram).

$\mathrm{R}$ : $\quad$ So, we cannot classify it as a negative complex number?

R2 : That is what I think la...

Respondent 2 grasped the concept of complex numbers. She was able to logically analyse the complex number and came up with a conclusion that there were no such thing as negative complex numbers. She tried to retrieve her memory in working with complex numbers and she found out that she never met the notion of negative complex numbers. This indicated that she was not affected by her experience in working with real numbers. As a result, she was not interfered by any supportive conceptions or problematic conceptions that might have arisen from the context of real numbers. She was able to draw that conclusion, through graphical method which showed that a complex number was actually a point on a complex plane, and thus, she concluded that it was not logical to call a point on a complex plane as a negative number.

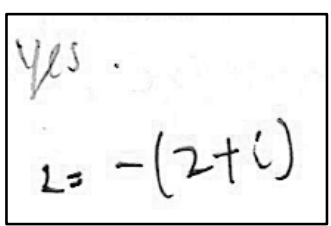

Figure 3: Respondent 3(Item 7) 
$\mathrm{R}$ : $\quad$ Do you think that $\mathrm{z}$ is a negative complex number?

R2 : Yes. It's negative because the negative can be factored out right? (writing $-(2+2 i)$ ).

$\mathrm{R}$ : $\quad$ So for you $\mathrm{z}$ is a negative complex number?

R2 : Yes I think so. Based on my logic, yes, I think so.

Respondent 3 had a problematic conception that originated from his experience in working with real numbers. He reasoned the idea of negative complex numbers by factorizing the negative sign as shown in Figure 3.Based on the appearance of $-(2+i)$, it is similar to the appearance of negative numbers in real numbers context such as $-a$ where $a$ is a positive real number. This problematic conception arises from the context of real numbers. However, the issue was Respondent 3 wasn't aware of this problematic conception and he interpreted this problematic conception as a supportive conception thus he came up with his notion of negative complex numbers that was incorrect.

Item 10 in the questionnaire asked "Do you think that $2+3 i<4+3 i$ ? Explain.”

$$
\text { Yes. } 4 \text { is bigger than? }
$$

Figure 4: Respondent 1 (Item 10)

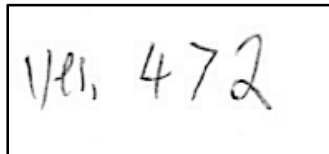

Figure 5: Respondent 3 (Item 10)

Respondent 1 and Respondent 3 agreed with the given inequalities. They were thinking as if 3iwas a real variable and can be eliminated or ignored. Treating $3 i$ as if it was a real variable may be considered as a problematic conception that arises from the real number context however the respondents were not aware of this problematic conception. They further elaborated:

“...because the $3 \mathrm{i}$ is the same for both complex numbers, we just compare the 2 and 4."
While Respondent 1 and 3 agreed to the given inequalities, Respondent 2 disagreed with them. Below is her reasoning:

$$
\begin{aligned}
& \text { No. We can't conclude it like this, we can compare } \\
& \text { their } g \text { arg } 2 \text { and distance. }
\end{aligned}
$$

Figure 6: Respondent 2 (Item 10)

Respondent 2 said that two different complex numbers should be compared in terms of angle (Argument Z) and distance from the origin. She didn't have any past experiences in ordering two complex numbers. Therefore, she felt that complex numbers cannot be ordered. Clearly, she was referring to her experience in working with complex numbers thus she was not affected by any supportive conceptions or problematic conceptions that may have arisen from other contexts.

Item 11 asked "Is the working shown correct? Explain."

$$
\begin{aligned}
& \frac{2}{i}=\frac{\sqrt{4}}{\sqrt{-1}}=\sqrt{\frac{4}{-1}} \\
& =\sqrt{-4}=\sqrt{-1} \sqrt{4} \\
& =2 i \\
& \text { Is the working shown above CORRECT? Explain }
\end{aligned}
$$

Figure 7: Item 11

All the three respondents agreed that the working shown above was correct. This was interesting because in real numbers we can always combine the radicals of numerator and denominator. Take for instance, $\frac{\sqrt{5}}{\sqrt{6}}=\sqrt{\frac{5}{6}}$. However, this is not always true in complex numbers. Clearly, this was a problematic conception that originated from working with real numbers that impeded the sense making of complex numbers. Below are the responses from each respondent:

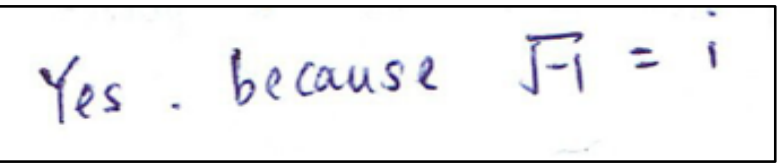

Figure 8: Respondent 1 (Item 11)

$$
\text { Yes, hasedon my linowledge, His make sence. }
$$

Figure 9: Respondent 3 (Item 11) 
Incorrect. $\frac{2}{i}$ impossible equal to $\partial i$.

Figure 10: Respondent 2 (Item 11)

Although Respondent 2responded that the working shown was incorrect, the follow-up interview revealed that she had some uncertainties. The interview excerpt below provides further details.

R : What do you think about this? (pointing to question 11). Can we combine this (Pointing at the square root)?

R2 : Can... can combine.

$\mathrm{R}$ : Where have you learnt this before? Where have you seen this operation?

R2: Mathematics.

$\mathrm{R}$ : $\quad$ Have you ever done this before where we combine the square roots?

R2: Yes. Combined the square root before but I haven't tried to combine the ... with $i$ before.

$\mathrm{R}$ : $\quad$ So, because $i$ is equal to square root of negative one right?

R2: Yes.

$\mathrm{R}$ : $\quad$ So, (pointing on the working shown in question 11) on the top, 2 is equal square root of four. So do you think that it is logical for us errr... can we combine it? What do you think?

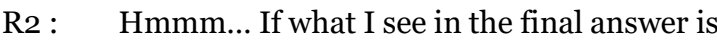
like this la...

$\mathrm{R}$ : If we omit the final answer first (cover the answer up). If we look at it like this, let say you don't know the final answer. Do you think we can combine into $\sqrt{\frac{4}{-1}}$ ?

R2: Yes. We can.

$\mathrm{R}$ : Meaning to say that after you saw the final answer, you make a conclusion that this is incorrect? Is it?

R2 : Yes.

In the follow-up interview, Respondent 2suspected the given working was incorrect by focusing on the final answer She assumed that it was fine to combine the radicals (as shown in Figure 7) because of her experience in working with the radicals in real numbers context. Even though she has never tried to combine radicals with $i$ before however she knew that $i^{2}=-1$ and -1 was a real number thus she concluded that the working was correct. If we reflect further, we can see that $i^{2}=\sqrt{-1} \times \sqrt{-1}=\sqrt{(-1)^{2}}=-1$ and superficially it seems fine to combine radicals in multiplication.

Item 17 asked "Two complex numbers $z=2+3 i$ and $u=6+2 i$. Find the value of $z+u$."

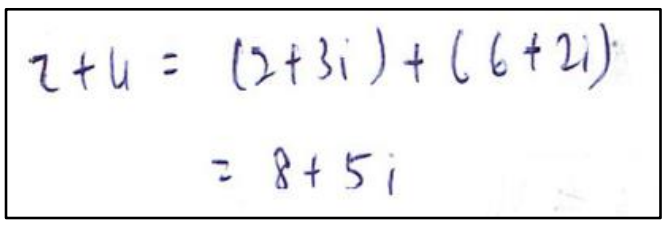

Figure 11: Respondent 1(Item 17)

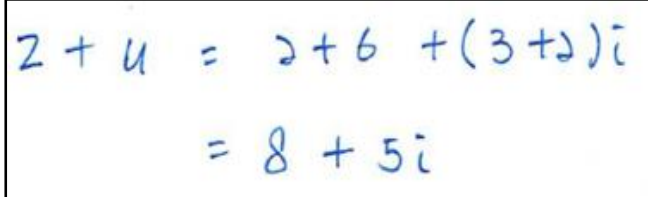

Figure 12: Respondent 2(Item 17)

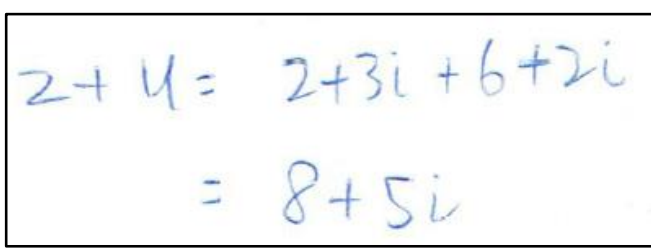

Figure 13: Respondent 3 (Item 17)

According to Figures 11, 12 and 13, all three respondents managed to solve the given mathematical item successfully. All of them agreed that their prior experience in learning the addition of real variables helped them to make sense the addition of two complex numbers. In this case, they were treating the imaginary number, $i$ as if it was a real variable. This was a supportive conception that arose from the real number context. Below is the interview transcript of Respondent 1:

R: Do you have problems in doing this calculation?

R1: No.

$\mathrm{R}$ : Why? Can you explain?

R1 : What I have learnt before is when there are two complex numbers, then real plus real and imaginary plus imaginary. Then for this question I get $8+5$ i.

$\mathrm{R}$ : $\quad$ So in this case, the " $i$ " behaves like a real 
variable?

R1 : Yes. I think they are the same concept. Like $(2 x+3 y)+(3 x+5 y)$. So, we get $5 x+8 y$. So, the same concept.

R : $\quad$ So, the concept you learnt before in algebra helps you to understand the addition of complex numbers?

R1: Yes.

Item 18 asked "Two complex numbers $z=2-4 i$ and $u=3+2 i$. Find the value of $z \cdot u$.”

$$
\begin{aligned}
z \cdot u & =(2-4 i)(3+2 i) \\
& =6+4 i-12 i-8 i^{2} \\
& =6-8 i-8(-1) \\
& =6-8 i+8 \\
& =14-8 i
\end{aligned}
$$

Figure 14: Respondent 1 (Item 18)

$$
\begin{aligned}
z \cdot u & =(2-4 i)(3+2 i) \\
& =6+4 i-12 i-8 i^{2} \\
& =6 \neq-8 i+8 \\
& =14-8 i
\end{aligned}
$$

Figure 15: Respondent 2(Item 18)

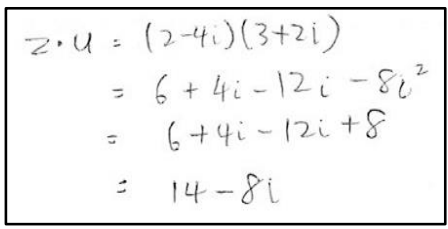

Figure 16: Respondent 3(Item 18)

Based on Figures 14, 15 and 16, we can notice that the respondents had no problem in performing the multiplication of two complex numbers. Again, the respondents had supportive conceptions that arose from real numbers context which enabled them to find the answer easily. They further elaborated that:

$\mathrm{R}$ : This is multiplication between complex numbers. Is the expansion works the same way as what you have learnt in previous algebra classes?
R2: Mmm... Yes, you just assume that $i$ is an unknown, so we can get this answer (pointing to her working) then because is quare is equal to -1 , soi times (pointing at 8) with -1 so I can get this(pointing at +8 in her working) so I finally did get $14-8 \mathrm{i}$.

$\mathrm{R}$ : Okay, so what you have learnt before in algebra that helps you to understand the process of multiplication of complex numbers?

R2 : Yes.

Obviously, the respondents' past experience in dealing with real variables multiplication supports their learning of complex numbers.

\section{CONCLUSION}

The data shows that there are several problematic conceptions that stem from real numbers context have affected the learning of complex numbers of the respondents. The three respondents tried to make sense of a new context by referring to their prior experience in real numbers. For example, in Item 7 and Item 10, Respondents 1 and 3 both had problematic conceptions in reasoning negative complex numbers and the existence of order in complex numbers. On the other hand, Respondent 2 referred to her past experience in working with complex numbers that enabled her to notice that the notions of negative numbers and inequality do not extend to complex numbers. Item 11 shows that the respondents were heavily affected by their experience in working with real numbers that led them to think that it was fine to combine the radicals. They didn't aware this problematic conception. This study discovers some supportive conceptions in the learning of complex numbers that stem from real numbers context. In this case, the concepts of addition and multiplication of real variables are supportive in the learning of complex numbers. The findings of this study are consistent with Chin and Jiew (2018) that highlights how the learning in the context of real numbers may impede the sense making of complex numbers. In conclusion, based on the gathered data, we can see that the respondents reasoned a new situation based on their prior knowledge or past experiences. It would be beneficial to explore further on how humans make sense of mathematics so that teachers can help them better. 


\section{REFERENCES}

Bromley, D. B. 1986, The Case-study Method in Psychology and Related Disciplines. Chichester: John Wiley \& Sons.

Bromley, D. B. 1990, Academic contributions to psychological counselling. 1. A philosophy of science for the study of individual cases. Counselling Psychology Quarterly, 3(3), 299-308. https://doi.org/10.1080/09515079008254261

Chin, K. E. 2013, Making sense of mathematics: supportive and problematic conceptions with special reference to trigonometry (Unpublished doctoral thesis). Retrieved from http://wrap.warwick.ac.uk/58403

Chin, K. E. 2014, Supportive and problematic aspects in mathematical thinking over the longer term. In S. Oesterle, C. Nicol, P. Liljedahl, P., \& D. Allan (Eds.), Proceedings of the 38th Conference of the International Group for the Psychology of Mathematics Education and the 36th Conference of the North American Chapter of the Psychology of Mathematics Education, 6, 41.

Chin, K. E., \&Jiew, F. F. 2018, A framework for making sense of mathematics. In F. J. Hsieh (Ed.), Proceedings of the 8th ICMI-East Asia Regional Conference on Mathematics Education, 2, 309-319.

Chin, K. E., \&Jiew, F. F. (2019). Changes of meanings in multiplication across different contexts: The case of Amy and Beth. Eurasia Journal of Mathematics, Science and Technology Education, 15(8), em1739. https://doi.org/10.29333/ejmste/108440
Chin, K. E., \& Pierce, R. 2019, University students' conceptions of mathematical symbols and expressions. Eurasia Journal of Mathematics, Science and Technology Education, 15(9), em1748. https://doi.org/10.29333/ejmste/103736

Chin, K. E., \& Tall, D. O. 2012, Making sense of mathematics through perception, operation and reason: The case of trigonometric functions. In Tai-Yih Tso (Ed.), Proceedings of the 36th Conference of the International Group for the Psychology of Mathematics Education, 4, 264.

Danenhower, P. 2000, Teaching and learning complex analysis at two British Columbia Universities. (Unpublished Doctoral thesis). Retrieved from http://www.collectionscanada.gc.ca/obj/S1/f2/dsk1/tape3 /PQDD_ooo8/NQ61636.pdf

Elizabeth Conner, M., Rasmussen, C., Zandieh, M., \& Smith, M. 2007, Student understanding of complex numbers. Electronic Proceedings for the Tenth Special Interest Group of the Mathematical Association of America on Research in Undergraduate Mathematics Education, Conference on Research in Undergraduate Mathematics Education. $\quad$ Retrieved from http://sigmaa.maa.org/rume/crume2007/papers/connerrasmussen-zandieh-smith.pdf 